Even faster and even more accurate first-passage time densities and distributions for the Wiener diffusion model

Gondan, Matthias; Blurton, Steven Paul; Kesselmeier, Miriam

Published in:

Journal of Mathematical Psychology

DOI:

10.1016/j.jmp.2014.05.002

Publication date:

2014

Document version

Publisher's PDF, also known as Version of record

Citation for published version (APA):

Gondan, M., Blurton, S. P., \& Kesselmeier, M. (2014). Even faster and even more accurate first-passage time densities and distributions for the Wiener diffusion model. Journal of Mathematical Psychology, 60, 20-22. https://doi.org/10.1016/j.jmp.2014.05.002 
Notes and comment

\title{
Even faster and even more accurate first-passage time densities and distributions for the Wiener diffusion model ${ }^{\star \Delta}$
}

\author{
Matthias Gondan $^{\mathrm{a}, *}$, Steven P. Blurton ${ }^{\mathrm{b}}$, Miriam Kesselmeier $^{\mathrm{c}}$ \\ a Department of Psychology, University of Copenhagen, Denmark \\ ${ }^{\mathrm{b}}$ Institute for Psychology, University of Regensburg, Germany

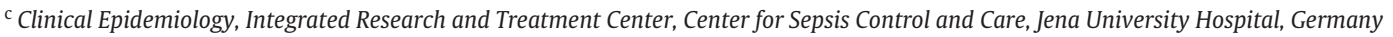

\section{H I G H L I G H T S}

- Infinite series describe the first passage times in the Wiener diffusion model.

- We provide efficient formulae for the density and distribution of first passages.

- An implementation in R statistical language is included in the online appendix.

\section{A R T I C L E I N F O}

\section{Article history:}

Received 31 January 2014

Received in revised form

15 May 2014

\section{Keywords:}

Diffusion model

Wiener process

First passage times

Response times

\begin{abstract}
A B S T R A C T
The Wiener diffusion model with two absorbing barriers is often used to describe response times and error probabilities in two-choice decisions. Different representations exist for the density and cumulative distribution of first-passage times, all including infinite series, but with different convergence for small and large times. We present a method that controls the approximation error of the small-time representation that occurs due to finite truncation of these series. Our approach improves and simplifies related work by Navarro and Fuss (2009) and Blurton et al. (2012, both in the Journal of Mathematical Psychology). (C) 2014 Elsevier Inc. All rights reserved.
\end{abstract}

\section{Introduction}

In the presence of two mutually exclusive outcomes, event times are often described by time-homogeneous Brownian motion drifting between two absorbing barriers (Wiener diffusion model, e.g., Busemeyer \& Townsend, 1993; Diederich, 1997; Ratcliff, 1978). For example, in two-choice decisions, a hidden underlying state is assumed to randomly move between two alternatives until one of two decision criteria is reached. For given parameters, the model predicts the probabilities for the two choices as well as the time it takes until the decision is made.

In a typical parametrization, a Wiener process $\mathbf{X}(t)$ starts at $\mathbf{X}(0)=z$ and moves randomly with drift $v$ and variance $\sigma^{2}=1$

\footnotetext{
Supported by the German Research Foundation (DFG, Grant GR 988/20-2 to Mark W. Greenlee and Matthias Gondan). A script written in R statistical language ( $\mathrm{R}$ Core Team, 2014) is provided as online supplemental material (see Appendix A).

* Correspondence to: Department of Psychology, University of Copenhagen, Øster Farimagsgade 2A, 1353 København, Denmark.

E-mail address: Matthias.Gondan@psy.ku.dk (M. Gondan).
}

between two absorbing barriers at 0 and $a, 0<z<a$. For convenience, define $w=z / a$ so that $0<w<1$. We consider the density $f^{0}$ and distribution $F^{0}$ of first-passage times at the lower barrier. The density of first passages at the upper barrier is given by $f^{a}(t \mid v, a, w)=f^{0}(t \mid-v, a, 1-w)$. For non-unit variance, the density at the lower barrier is given by $f^{0}(t \mid v / \sigma, a / \sigma, w)$. The same relations hold for the distribution function.

\section{Density and distribution of first-passage times}

The density of first-passage times can be factorized and thus be reduced to a standard form with one parameter (Navarro \& Fuss, 2009, Eq. 2),

$$
f^{0}(t \mid v, a, w)=\frac{1}{a^{2}} \exp \left(-v a w-\frac{v^{2} t}{2}\right) \cdot f^{0}\left(\frac{t}{a^{2}} \mid 0,1, w\right)
$$

with $t>0$. Hereinafter, $f(t)$ shall denote the one-parameter density. Two representations exist for $f(t)$ that show different behavior. The "large-time representation" $f_{\ell}$ shows good convergence for 
large $t$ (e.g., Ratcliff, 1978),

$f_{\ell}(t)=\pi \sum_{k=1}^{\infty} k \exp \left(-\frac{k^{2} \pi^{2} t}{2}\right) \sin (k \pi w)$,

whereas the "small-time representation" $f_{s}$ converges quickly for small $t$ (e.g., Van Zandt, Colonius, \& Proctor, 2000),

$f_{s}(t)=t^{-3 / 2} \sum_{k=-\infty}^{\infty}(w+2 k) \phi\left(\frac{w+2 k}{\sqrt{t}}\right)$

with $\phi$ denoting the standard normal density.

The terms of both series vanish for large $|k|$. Navarro and Fuss (2009) determined upper bounds for the approximation error that results from finite truncation of the terms with $|k|>K$ and provided expressions for the minimum number of terms $K_{\ell}$ and $K_{s}$ that assure a predefined level of accuracy $\varepsilon$ in the two representations. By comparing $K_{\ell}$ with $K_{s}$, and taking into account the calculations involved in $f_{\ell}$ and $f_{s}$, the representation with the least computational effort can be chosen.

Similar expressions exist for the cumulative distribution of first-passage times which is often used in model fitting. For the large-time representation $F_{\ell}(t)$ see, for example, Eq. (A12) in Ratcliff (1978). Here we consider only the small-time representation (e.g., Horrocks \& Thompson, 2004; Van Zandt et al., 2000),

$$
\begin{aligned}
& F_{S}(t)=P-\operatorname{sgn} v \\
& \quad \cdot \sum_{k=-\infty}^{\infty}\left[\exp (-2 v a k-2 v a w) \Phi\left(\operatorname{sgn} v \cdot \frac{2 a k+a w-v t}{\sqrt{t}}\right)\right. \\
& \left.\quad-\exp (2 v a k) \Phi\left(\operatorname{sgn} v \cdot \frac{-2 a k-a w-v t}{\sqrt{t}}\right)\right]
\end{aligned}
$$

with $P=\frac{\exp (-2 v a w)-\exp (-2 v a)}{1-\exp (-2 v a)}$ denoting the total probability of absorption at the lower barrier and $\Phi$ the standard normal distribution. The above expressions for $F_{S}(t)$ and $P$ are undefined for zero drift, for which simplified expressions can be found. Using a very similar approach as Navarro and Fuss (2009), Blurton, Kesselmeier, and Gondan (2012) proposed an efficient way for the approximation of $F_{S}(t)$ and $F_{\ell}(t)$ that assures a pre-specified precision $\varepsilon$.

Within this error bound, both approaches (Blurton et al., 2012; Navarro \& Fuss, 2009) yield a smooth surface for model fitting (e.g., in likelihood maximization or in goodness-of-fit optimization), at a computing speed equal to, and in many cases superior to standard truncation methods. In both approaches, the total truncation error is controlled by determining an upper bound for the sum of the truncated terms with standard calculus tools.

\section{Improved small-time representations}

In both representations, controlling the approximation error requires the determination of an upper bound for an infinite sum of truncated terms which might be very conservative. In addition, Expression (2) for $F_{s}(t)$ requires an approximation of $\exp (y) \Phi(-x)$ at large $x$ and $y$ (Kiani, Panaretos, Psarakis, \& Saleem, 2008), and suffers from computational and numerical problems at drift rates near zero.

It turns out that $F_{s}$ can be restated using the reciprocal hazard rate ("Mill's ratio") of the Normal distribution $M(x)=\frac{1-\Phi(x)}{\phi(x)}$ (Hall, 1997, p. 347), resulting in

$$
\begin{aligned}
F_{S}(t)= & \exp \left(-v a w-\frac{v^{2} t}{2}\right) \sum_{j=0}^{\infty}(-1)^{j} \phi\left(\frac{r_{j}}{\sqrt{t}}\right) \\
& \times\left[M\left(\frac{r_{j}-v t}{\sqrt{t}}\right)+M\left(\frac{r_{j}+v t}{\sqrt{t}}\right)\right]
\end{aligned}
$$

with $r_{j}=j a+a w$ for even $j$, and $r_{j}=j a+a(1-w)$ for odd $j$, so that $r_{j}<r_{j^{\prime}}$ for $j<j^{\prime}$. The equivalence of the two representations is shown in the online supplementary material (see Appendix A).

Because of $(-1)^{j}$, and for positive arguments of $\phi$ and $M$ (see below), Expression (3) is an absolutely decreasing alternating series. The absolute truncation error is then always smaller than the first truncated term. In other words, evaluation of the series can be stopped as soon as the absolute value of the jth term is below $\varepsilon$.

Because the even and the odd terms are slightly differently defined, and to further reduce the truncation error, the practical implementation of (3) will add up $K$ pairwise differences of even and odd terms from $j=0$ to $2 K-1$. Denote the first truncated (even) term in (3) by

$$
\begin{aligned}
s_{2 K}= & \exp \left(-v a w-\frac{v^{2} t}{2}\right) \phi\left(\frac{r_{2 K}}{\sqrt{t}}\right) \\
& \times\left[M\left(\frac{r_{2 K}-v t}{\sqrt{t}}\right)+M\left(\frac{r_{2 K}+v t}{\sqrt{t}}\right)\right] .
\end{aligned}
$$

Then, consider $s_{2 K}^{\prime} \geq s_{2 K}$ in which the numerators of the arguments of $\phi$ and $M$ are replaced by $r_{2 K}-|v| t$. Simplifications lead to

$s_{2 K}^{\prime}=2 \exp \left(-v a w-\frac{v^{2} t}{2}\right)\left[1-\Phi\left(\frac{r_{2 K}-|v| t}{\sqrt{t}}\right)\right]$.

This upper bound $s_{2 K}^{\prime}$ is below $\varepsilon$ if

$$
\begin{aligned}
K \geq & \frac{\sqrt{t}}{2 a} \cdot \Phi^{-1}\left[1-\frac{1}{2} \exp \left(v a w+\frac{v^{2} t}{2}+\log \varepsilon\right)\right] \\
& +\frac{|v| t-a w}{2 a}
\end{aligned}
$$

which also keeps the total truncation error below $\varepsilon$. Positivity of the arguments of $\phi$ and $M$ is given for $K \geq \frac{|v| t-a w}{2 a}$.

We now consider the first-passage density in its simplified oneparameter form with zero drift (1).

$f_{s}(t \mid 0,1, w)=\frac{1}{\sqrt{2 \pi t^{3}}} \sum_{j=-\infty}^{\infty}(w+2 j) \exp \left[-\frac{(w+2 j)^{2}}{2 t}\right]$.

For $j \geq J$ with $|w+2 J| \geq \sqrt{2 t}$, the decrease of the exponential dominates the increase of the factor, so that $f_{s}$ is again recognized as an absolutely decreasing alternating series. Let $s_{j}$ denote individual terms of (5). Then, $\left|s_{-J}\right|>\left|s_{J}\right|>\left|s_{-(J+1)}\right|>\left|s_{J+1}\right|$, and so on, with an alternating sign. Reordered this way, evaluation of (5) can stop if $j \geq J$ and the absolute value of the next term is below $\varepsilon$.

For the practical implementation it seems again reasonable to accumulate $K$ pairs $\left(s_{0}>0, s_{-1}<0\right)$, then $\left(s_{1}, s_{-2}\right)$, and so on, until $\left(s_{K-1}, s_{-K}\right)$. The minimum number of pairs $K$ is then given by $K \geq J$ and

$K \geq \frac{1}{2} \cdot \sqrt{-t\left(u_{\varepsilon}-\sqrt{-2 u_{\varepsilon}-2}\right)}-w / 2$

with $u_{\varepsilon}=\log \left(2 \pi t^{2} \varepsilon^{2}\right)$ (for a stepwise derivation, see the online supplemental material, Appendix A). The criterion is obtained by taking the square on both sides of $s_{K} \leq \varepsilon$ and applying Lambert's $W$ function to the result that has the form $x \exp (x) \geq y$. A conservative bound for the lower branch of $W$ is given by Chatzigeorgiou (2013, Theorem 1).

The above derivation holds for drift $v=0$ and barrier separation $a=1$. For the general case (1), $K$ has to be determined using a modified convergence criterion $\varepsilon^{\prime}=\varepsilon \cdot a^{2} \exp \left(v a w+\frac{v^{2} t}{2}\right)$.

\section{Discussion}

The present note provides improved finite approximations of the small-time representations of the density and distribution of first-passages in the two-barrier diffusion model. By comparing the required number of iterations in the small-time representations 

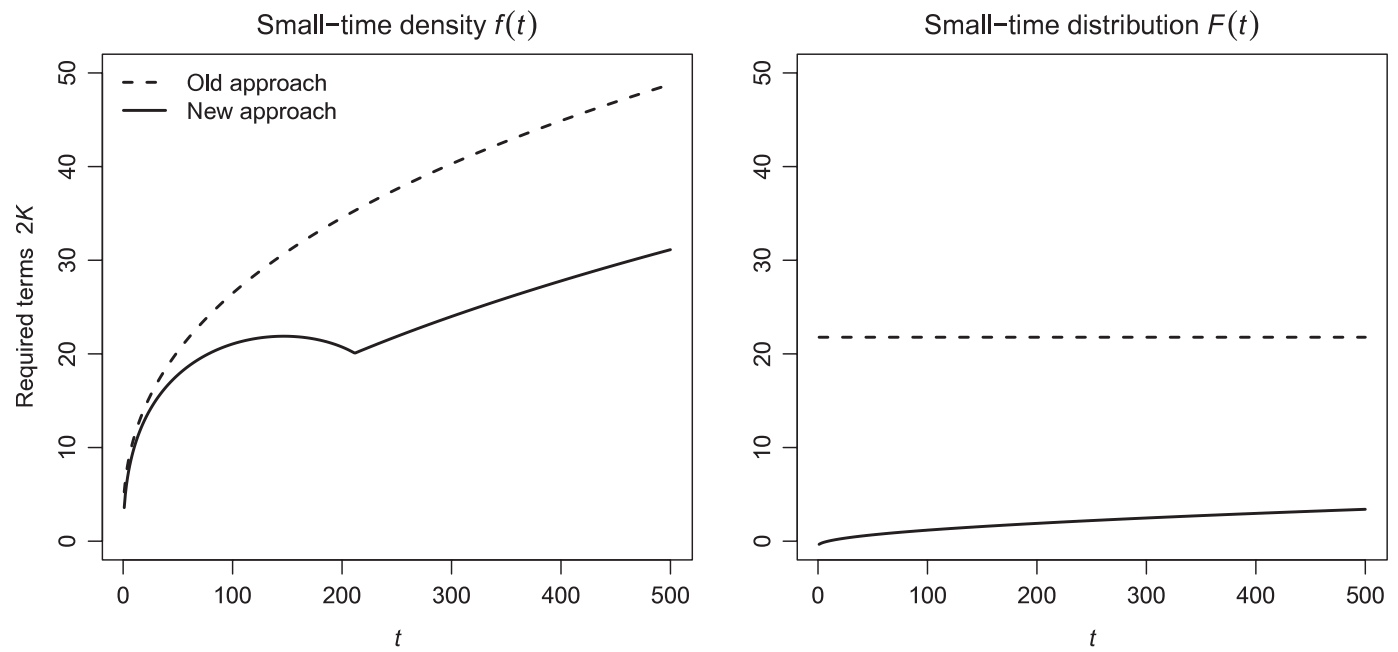

Fig. 1. Improved criteria for the small-time representations in the Wiener diffusion model. The figures show the number of terms that are required to reach a precision of $\varepsilon=0.001$ in the series for the density and distribution as a function of $t$ at which $f$ and $F$ are evaluated. These numbers are driven by the index of the term at which the series starts to decrease, and by the index of the first term that is below $\varepsilon$. In the density function, the first criterion $(K \geq J)$ dominates the second criterion for small $t$ (6), causing a non-monotone relation between $t$ and $2 K$. Compared to the previous approaches, the required number of terms is slightly reduced for the first-passage time density (left, $f$ with $w=0.5$ ) and considerably reduced for the distribution (right, $F$ with $v=0.01, a=20, w=0.5$ ).

with their large-time counterparts (Blurton et al., 2012; Navarro \& Fuss, 2009), and adjusting for the time necessary to evaluate a single term of the series, the representation which requires least computational effort can be chosen.

Compared to previous approaches, the improvement is fourfold: Restating the expressions for the density and distribution as absolutely decreasing alternating series, the derivation of the truncation error for (3) and (5) is considerably simplified, because only a single term needs to be controlled instead of an infinite sum. As a consequence, the estimate for the actual approximation error is improved, and fewer terms are needed to obtain a prespecified precision (Fig. 1). Because efficient implementations exist for Mill's ratio (erfcx in Matlab, zeta in R-package sn, Azzalini, 2014), and $M(x)$ behaves well for large $x$, the distribution function (3) is numerically less problematic than the standard form with $\exp (y) \Phi(-x)$ that needs an approximation for large $x$ and $y$ (see Blurton et al., 2012, supplemental material). Finally, the expressions for the distribution function are well-defined for all drift rates including $v=0$ and rates close to zero.

Because of standard convergence properties of decreasing alternating series, standard ad hoc approaches are actually justified that stop evaluation as soon as a single term is below the tolerance-this holds at least for the small-time representations (we did not find a respective solution for the large-time representation). Because two alternative representations exist for both the density and the distribution, and because it is numerically more precise to accumulate sums from small to large terms, it is still advantageous to determine, in advance, the required number of terms, and we provide improved estimates for this number to attain a pre-specified tolerance (Ineqs. (4) and (6)). For the large-time counterparts, we refer to the solutions already offered by Navarro and Fuss (2009) and Blurton et al. (2012). Then, one can decide in advance which representation to choose for optimal numerical properties and minimal computational costs.

\section{Appendix A. Supplementary material}

Supplementary material related to this article can be found online at http://dx.doi.org/10.1016/j.jmp.2014.05.002. The supplementary material includes more detailed derivations and an implementation in R (R Core Team, 2014).

\section{References}

Azzalini, A. (2014). The R sn package: the skew-normal and skew- $t$ distributions (version 1.0-0). Università di Padova, Italia.

Blurton, S. P., Kesselmeier, M., \& Gondan, M. (2012). Fast and accurate calculations for cumulative first-passage time distributions in Wiener diffusion models. Journal of Mathematical Psychology, 56, 470-475.

Busemeyer, J. R., \& Townsend, J. T. (1993). Decision field theory: a dynamiccognitive approach to decision making in an uncertain environment. Psychological Review, 100, 432-459.

Chatzigeorgiou, I. (2013). Bounds on the Lambert function and their application to the outage analysis of user cooperation. IEEE Communications Letters, 17, $1505-1508$.

Diederich, A. (1997). Dynamic stochastic models for decision making under time constraints. Journal of Mathematical Psychology, 41, 260-274.

Hall, W. J. (1997). The distribution of Brownian motion on linear stopping boundaries. Sequential Analysis, 16, 345-352

Horrocks, J., \& Thompson, M. E. (2004). Modeling event times with multiple outcomes using the Wiener process with drift. Lifetime Data Analysis, 10, 29-49.

Kiani, M., Panaretos, J., Psarakis, S., \& Saleem, M. (2008). Approximations to the normal distribution function and an extended table for the mean range of the normal variables. Journal of the Iranian Statistical Society. (JIRSS), 7, 57-72.

Navarro, D. J., \& Fuss, I. G. (2009). Fast and accurate calculations for first-passage times in Wiener diffusion models. Journal of Mathematical Psychology, 53, 222-230.

R Core Team (2014). R: A Language and Environment for Statistical Computing. Vienna, Austria: R Foundation for Statistical Computing.

Ratcliff, R. (1978). A theory of memory retrieval. Psychological Review, 85, 59-108.

Van Zandt, T., Colonius, H., \& Proctor, R. W. (2000). A comparison of two response time models applied to perceptual matching. Psychonomic Bulletin E Review, 7, 208-256. 


\section{Online Supplement}

This is the online supplemental material for "Even faster and even more accurate first-passage time densities and distributions for the Wiener diffusion model" by Gondan, Blurton, and Kesselmeier. The online supplement provides details on the derived criterion for convergence as well as some example code in the $\mathrm{R}$ statistical language (R Core Team, 2014) as a separate file. In addition, we show the equivalence of the expressions for the small-time distribution in Hall (1997) and Blurton, Kesselmeier and Gondan (2012).

\section{Equivalence of the two small-time distributions}

Expression (3) yields the small-time representation of the distribution of first-passage times (Hall, 1997). In order to demonstrate its equivalence with the standard form (used, e.g., in Blurton et al., 2012), we treat the positive and negative terms separately:

$$
\begin{gathered}
F_{s}(t)=\exp \left(-v a w-\frac{v^{2} t}{2}\right) \sum_{k=0}^{\infty}\left\{\phi\left(\frac{r_{2 k}}{\sqrt{t}}\right)\left[M\left(\frac{r_{2 k}-v t}{\sqrt{t}}\right)+M\left(\frac{r_{2 k}+v t}{\sqrt{t}}\right)\right]\right. \\
\left.-\phi\left(\frac{r_{2 k+1}^{\prime}}{\sqrt{t}}\right)\left[M\left(\frac{r_{2 k+1}^{\prime}-v t}{\sqrt{t}}\right)+M\left(\frac{r_{2 k+1}^{\prime}+v t}{\sqrt{t}}\right)\right]\right\}
\end{gathered}
$$

with $r_{2 k}=2 a k+a w$ for the positive terms (even $j$ in Expr. 3), and $r_{2 k+1}^{\prime}=$ $2 a k+2 a-a w$ for the negative terms (odd $j$ ).

For the Normal distribution, $\phi(x)=\frac{1}{\sqrt{2 \pi}} \exp \left(-\frac{x^{2}}{2}\right)$, and "Mill's ratio" is $M(x)=\sqrt{\frac{\pi}{2}} \exp \left(\frac{x^{2}}{2}\right) \operatorname{erfc}\left(\frac{x}{\sqrt{2}}\right)$, with $\operatorname{erfc}\left(\frac{x}{\sqrt{2}}\right)=2[1-\Phi(x)]=2 \Phi(-x)$ denoting the "complementary error function". 
For positive drift, simplifications lead to

$$
\begin{aligned}
& F_{s}(t \mid v>0)=\exp (-v a w) \times \\
& \sum_{k=0}^{\infty}\left\{\exp \left(-r_{2 k} v\right)\left[1-\Phi\left(\frac{r_{2 k}-v t}{\sqrt{t}}\right)\right]+\exp \left(r_{2 k} v\right) \Phi\left(-\frac{r_{2 k}+v t}{\sqrt{t}}\right)\right. \\
& \left.\quad-\exp \left(-r_{2 k+1}^{\prime} v\right)\left[1-\Phi\left(\frac{r_{2 k+1}^{\prime}-v t}{\sqrt{t}}\right)\right]-\exp \left(r_{2 k+1}^{\prime} v\right) \Phi\left(-\frac{r_{2 k+1}^{\prime}+v t}{\sqrt{t}}\right)\right\}
\end{aligned}
$$

The difference of the two geometric series $\exp (-v a w) \sum_{k=0}^{\infty} \exp \left(-r_{2 k} v\right)$ and $\exp (-v a w) \sum_{k=0}^{\infty} \exp \left(-r_{2 k+1}^{\prime} v\right)$ corresponds to the total probability of absorption at the lower barrier, $P=\frac{\exp (-2 v a w)-\exp (-2 v a)}{1-\exp (-2 v a)}$. After replacing $r_{2 k}=2 a k+a w$ and $r_{2 k+1}^{\prime}=2 a(k+1)-a w$, the terms with $r$ and $r^{\prime}$ complement to two infinite series from $-\infty$ to $\infty$ :

$$
\begin{gathered}
F_{s}(t \mid v>0)=P-\sum_{k=-\infty}^{\infty}\left[\exp (-2 v a k-2 v a w) \Phi\left(\frac{2 a k+a w-v t}{\sqrt{t}}\right)\right. \\
\left.-\exp (2 v a k) \Phi\left(\frac{-2 a k-a w-v t}{\sqrt{t}}\right)\right]
\end{gathered}
$$

A similar argument can be made for $v<0$. The sgn-functions (e.g., in Blurton et al., 2012) summarize both positive and negative drift in a compact form.

\section{Criteria for the small-time density}

The series in (5) yields the small-time representation of the first-passage time density. Again, as argued in the text, for $K \geq \frac{\sqrt{2 t}-w}{2}$ it is a decreasing alternating series so that the truncation error is controlled if the first (positive) truncated term $s_{K}$ is below $\varepsilon$, i. e.

$$
\frac{w+2 K}{\sqrt{2 \pi t^{3}}} \exp \left[-\frac{(w+2 K)^{2}}{2 t}\right] \leq \varepsilon
$$


Taking the square on both sides (both sides are positive) and rearranging yields

$$
-\frac{(w+2 K)^{2}}{t} \exp \left[-\frac{(w+2 K)^{2}}{t}\right] \geq-2 \pi t^{2} \varepsilon^{2}
$$

which has the form $x \exp (x) \geq y$. The solution is obtained by applying Lambert's $W$ function to both sides (here the lower branch of Lambert's $W$ is needed),

$$
-\frac{(w+2 K)^{2}}{t} \leq W\left(-2 \pi t^{2} \varepsilon^{2}\right)
$$

Lambert's $W$ can either be determined numerically, which is computationally expensive. Alternatively, a lower bound is given by Chatzigeorgiou (2013, Theorem 1), $W[-\exp (u)]>u-\sqrt{-2 u-2}$. Setting $u_{\varepsilon}=$ $\log \left(2 \pi t^{2} \varepsilon^{2}\right)$, the above condition holds if

$$
-\frac{(w+2 K)^{2}}{t} \leq u_{\varepsilon}-\sqrt{-2 u_{\varepsilon}-2}
$$

which is then easily solved for $K$ and leads to

$$
K \geq \frac{1}{2} \cdot \sqrt{-t\left(u_{\varepsilon}-\sqrt{-2 u_{\varepsilon}-2}\right)}-w / 2
$$


evenfaster-r1.r

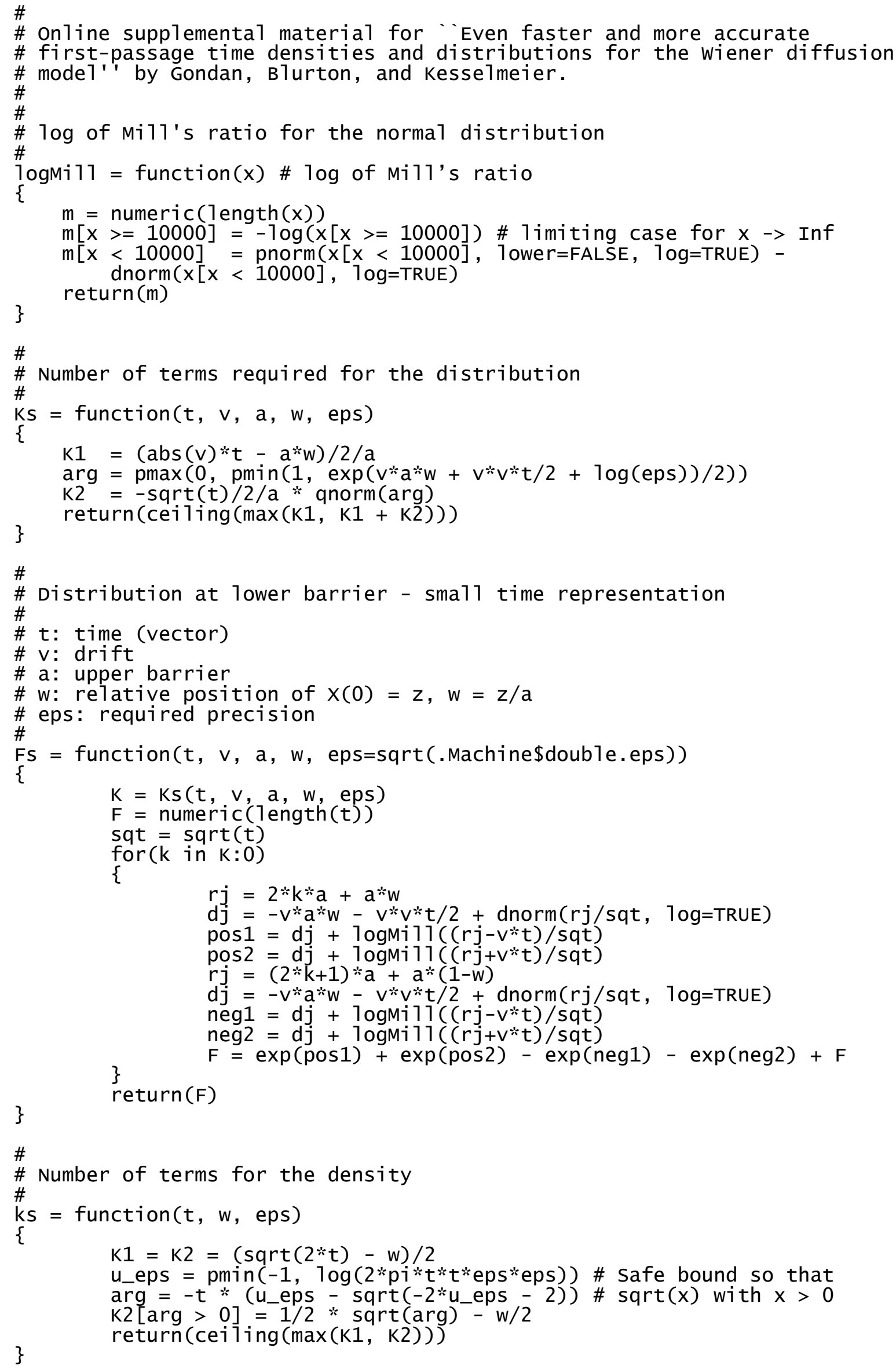


evenfaster-r1.r

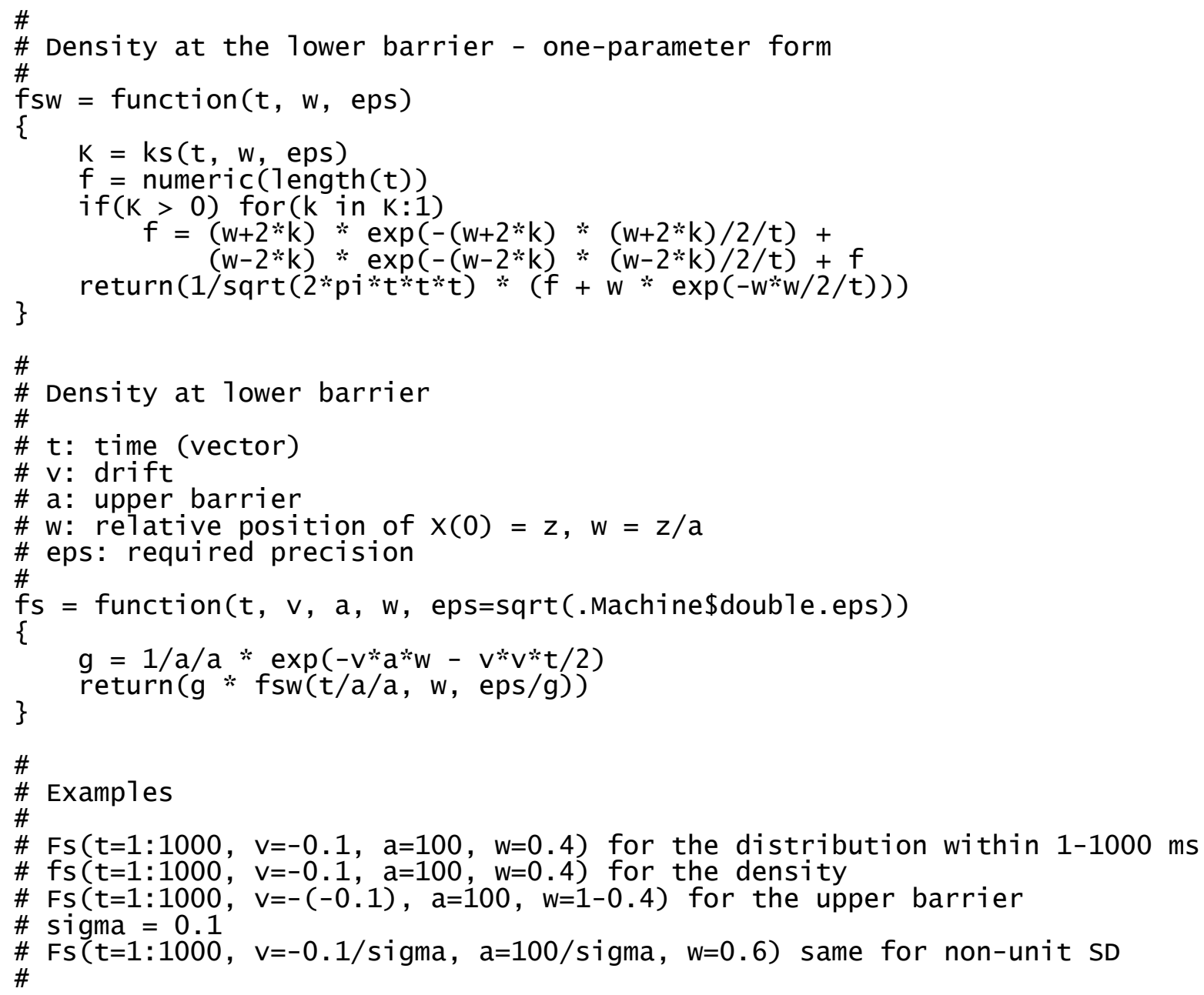

